\title{
Highlighting the Historical, Current, and Future Challenges to Control and Eradicate Malaria in Uganda - A Narrative Review
}

\author{
Article by Katwesige Wycliff \\ Public Health, Texila American University, Uganda \\ E-mail: katnagaish@yahoo.com
}

\begin{abstract}
Background: For the last century, malaria has caused a serious developmental setback to African countries. Historically, In Uganda, the control of malaria and its eradication challenges can be traced for more than 100 years ago especially the control and elimination activities connected to research and malaria control between1892 to 1949. During the early part of the 21st century, malaria received global health priority recognition until 1998 when the World Health organization (WHO) adopted a Rollback strategy. Currently, In May 2015, The World Health Organization (WHO) adopted a global Strategy for Malaria 2016-2030. This ambitious strategy apparently challenged by the fact that current tools are not adequate to achieve the planned targets. The future challenges to eradicate malaria are also real and need urgent solutions.

Methods: We searched Cochrane Libraries, EMBASE, Google Scholar, and unpublished ministry of health Uganda reports.

Objectives: To assess the historical, current and future challenges to malaria control and eradication and propose solutions to address them.

Results: Uganda has failed to eradicate malaria in the past century, due to challenges like lack of political commitment, limited resources for eradication programs, rampant history of antimalarial drug resistance, chronic and persistent antimalarial stock-outs in public facilities, resistance by parasites to ITNs and fragmentation in the coordination. Findings reveal that the past challenges still exist and are likely to last for more than a decade unless measures are in place to mitigate them. Uganda will face challenges in implementing newer effective interventions in future as well as the history of malaria eradication challenges may keep repeating itself.

Conclusion: The historical challenges in the eradication of malaria in Uganda are still affecting the current plans to eradicate malaria. Uganda may not eradicate malaria in next the15 years, basing on its current and future challenges ahead.
\end{abstract}

Keywords: Malaria, control, eradication, Uganda, challenges, Historical, current, Future.

\section{Introduction}

\section{Background}

Malaria has caused serious health and developmental challenges in the Sub-Saharan African region for several years. Although the disease is can both preventable and treatable, it continues to pose a great challenge to health and development, especially in the African continent that carries the largest burden of malaria globally. Despite tremendous achievements in control of malaria by the malaria-endemic countries between 2009-2010, nearly 800,000 child mortalities with 300 million episodes still occur annually (Schwartz, Brown, Genton, \& Moorthy, 2012). Public health interventions to control malaria are usually very cost-effective yielding highest returns on investment and yet only seven countries globally have managed to eliminate malaria in last 15years. The fight to control malaria is very prolonged while in some countries like Uganda slowed down by several crosscutting challenges. This review focuses on highlighting these challenges from the historical, current, and future perspectives. 
However, according to world malaria report 2015, the global risk and mortality due to malaria, remain very high. Over 254 million people in East and South Africa region are at a very high risk of disease, hence a global concern to accelerate current progress to check on morbidity and mortality in such countries (World malaria report 2015). According to Ministry of Health Uganda, Malaria ranks as one of the leading causes of morbidity and mortality in the country contributing to nearly $40 \%$ of all Outpatients clinic visits $(\mathrm{MOH}, 2011)$.The President's Malaria Initiative (PMI) along with the Global Fund are the main financial contributors to malaria control and eradication in Uganda.

Historically, the control of malaria can be traced for more than 100 years ago especially the control and elimination activities connected research and malaria control between 1892 to 1949. Control Malaria control can be defined as "All efforts to reduce malaria morbidity and mortality to a locally acceptable level through the use of preventive and curative measures" (The Roll Back Malaria Partnership, 2008b). In the early 1930s, WHO encouraged the control of malaria using mass spraying with Dichloro-Diphenyl-Trichloroethane (DDT).In line with 1948 WHO malaria eradication strategy, Uganda embarked on vector eradication interventions throughout the 1950s to 1990 (Akello, 2015). Meanwhile, in the early part of the 21st century, malaria control and eradication efforts became a wider part of the global health priorities. ("Global technical strategy for malaria 2016-2030," 2016) Until 1998, WHO adopted a Rollback strategy, including parasite based control interventions at the time, was most African countries were battling with rampant malaria epidemics leading to significant reduction in the spread of the disease. The interventions attracted large funding support to scale-up malaria control activities contributing to substantial reductions. WHO also envisaged a reduction of malaria incidence by $17 \%$ between the years 2000 to 2010 where it made a considerable success globally, but very limited success in Uganda (Cotter et al., 2013) The early diagnosis and management of malaria is currently strategized to control malaria in most African Countries. However, this strategy has faced challenges and failed to eradicate malaria in Uganda. Most health centers in Uganda are reported to have limited capacity in terms of human resource and infrastructural capacity to adequately implement universal parasite-based malaria diagnosis and treatment (Kyabayinze et al., 2012). Nowadays, World Health organization recommends artemisinin-based combination therapy (ACT) for treating uncomplicated Plasmodium falciparum malaria (Kassam, Collins, Liow, \& Rasool, 2015).

Currently, during the 2015 world malaria day, the success achieved with the invention of new techniques of prevention and treatment of malaria was highlighted (Hemingway et al., 2016). In 2016 the global technical strategy for next one and half decade likely to be strongly shaped by technological innovations in medicines, vaccines, and vector control as well as improved strategies for delivering commodities have been launched ("Global technical strategy for malaria 2016-2030," 2016). This review, therefore, highlights the key historical, current, and future challenges to malaria control in Uganda.

\section{The problem}

In May 2015, The World Health Organization (WHO) adopted a global Strategy for Malaria 2016-2030, that provides a framework for all malaria endemic countries to guide and support them attain full malaria control by reducing the incidence and mortality by $90 \%$ while achieving elimination in 35 countries by 2030. In the past century, Uganda has failed to eradicate malaria. The country's current health infrastructure remains incapacitated to implement the road map to eradication. Limited financial support and political commitment to eradicate malaria underpin the future fight against the disease to achieve elimination. Even though malaria treatment drugs are free in the public sector, a considerable proportion of Ugandans largely seek health services from the private sector. According to world malaria report 2013, the country malaria treatment policy is largely Artemether Lumenfantrin a as first line and Quinine for treatment of severe malaria (Rica \& Salvador, 2013). Resistance to the cost effective Quinine is likely to pose a great future challenge in Uganda especially when the country is struggling to procure more expensive artemisinin based combination with most 
of the recommended Intravenous Artesunate regularly out of stock almost yearly in most public facilities and resorting to only cheap and affordable Quinine. Uganda has a clear history of malaria drug resistance, especially to chloroquine and Fansidar (SP) which was cost effective, but the country seems not to learn from this history (Greenwood, 2014). Furthermore, there seem to be no plans to alternative drugs for future resistance to Quinine and Artemisinin-based combination in Uganda hence the need to inform researchers, and Malaria control programmers to plan for these challenges.

\section{Justification}

There is a global consensus that global elimination of Malaria is not possible using the current tools, hence a call for new evidence-based tools that will make it possible to achieve eliminations in most countries in the future. In Uganda, there seems to be no evidence to move from a controlled low-endemicity of malaria to total elimination despite having developed malaria elimination strategy by her malaria control program (Newby et al., 2016). Even if Uganda has registered a tremendous success in the control and eradication of malaria nationwide, the need to provide the evidence base to develop a feasible framework to achieve the country's 2030 international obligations is quite demanding. Such evidence can inform programming, increase political commitment, and national readiness to take on the ambitious tasks geared towards the elimination of malaria in Uganda. This review, therefore, is meant to assess and provide an insight of the historical, current and future challenges to control and eradicate malaria in Uganda.

\section{Findings}

\section{Historical challenges in control and eradication of malaria in uganda}

Malaria was in the past considered as a neglected disease. In the past, various theories were presented with the means by which malaria was transmitted. However, by 1898 the female anopheles mosquito was discovered by Dr. Ronald Ross as responsible for malaria parasite transmission (Olowe et al., 2015). In the early 1930s, WHO recommended the control of malaria by mass spraying with Dichloro-Diphenyl-Trichloroethane (DDT). There were three remarkable decades of great optimism after 1949. Most notably was the events characterized by this period especially, the 1950 Kampala Conference, the 1955 Global Malaria Eradication Program, the post-independence primary health care strategies for the African States in the 1960 and the Special research training Program on Tropical Diseases at the World Health Organization in 1975. It also took over 30years to re-examine antimalarial strategies (Alilio, Bygbjerg, \& Breman, 2004). In line with1948 WHO malaria eradication strategy, Uganda engendered tremendous effort to eradicate malaria by destroying the parasite using mass spraying campaigns using DDT in the 1950s and introduction of indoor residual spraying (IRS) in the 1960s (Kassam et al., 2015). Meanwhile, in the early part of the 21 st century, malaria received worldwide recognition as a priority global health issue ("Global technical strategy for malaria 2016-2030," 2016). Therefore, for Africa in general, tremendous achievements in control of malaria have been achieved, but fragmentation in the coordination remains a key challenge.

In the last 2 decades, Malaria in Uganda was managed with chloroquine monotherapy. Chloroquine (CQ), amodiaquine (AQ), and sulfadoxine-pyrimethamine (SP) used to be less expensive medicines, but the challenge was their treatment failure. The drug combinations meant to encounter treatment failure were proposed. Past evidence from the RCT showed that chloroquine (CQ) or Amodiaquine (AQ) plus Fansidar (SP) were the most cost effective option treatment combinations, but their effectiveness remained a challenge (Mcintosh, Kl, Mcintosh, \& Jones, 2010). The resistance to chloroquine prompted Uganda to change its treatment guidelines to use drug combinations like chloroquine and sulfadoxine Pyrimethamine (Fansidar). Malaria resistance was also reported in less than a decade promoting the adoption of the Artemisinin-based Combination Therapy (ACT). Most important to note is that Chloroquine was indeed a very cost effective drug, but no efforts 
were planned to prepare for its failure hence scientists in academia and pharmaceutical companies had to learn lessons (Greenwood, 2014).

In past 10years, the world resorted to massive vector control strategy with tremendous achievements as well as great advances in the fight to eradicate malaria with the malaria mortality being reduced to nearly $50 \%$ and related incidence by $50 \%$ (WHO, 2013).

\section{Current challenges of malaria control in uganda}

Malaria is currently among the major deadly diseases affecting the poor in Africa, causing avoidable mortality among children and women. Its toll is estimated to cause 881,000 mortalities annually with nearly $90 \%$ of the deaths happening in Sub-Saharan Africa, Uganda inclusive (Kokwaro, 2009). According to the WHO, Uganda still ranks third out of the 18 sub-Saharan African countries, after the Democratic Republic of Congo that account for 90 percent of P. Falciparum infections (WHO, 2014). The Uganda national malaria control program report confirms that the disease is ranked as the 6th highest in a number of annual mortality in Africa, as well as highest transmission rates globally with16 million cases and over 10,500 deaths reported in 2013 alone (Road, 2014). Recently the world malaria report 2013, revealed that Uganda relies mainly on ITNs, IRS, IPT, Larval control, early diagnosis, and treatment as well as surveillance to control and eliminate malaria (Rica \& Salvador, 2013). In another study, current and cost-effective interventions available include, effective vector control using LLINs and IRS, and timely effective treatment using ACT after a right diagnosis (Kokwaro, 2009). According to Akello, poverty is one of the current challenges to the control of malaria in the country (Akello, 2015). Other research shows that historically research on malaria control was focused on attacking the mosquito vectors and remains very significant intervention at our disposal. Since these parasites pass through the vector, current studies recognize that attacking the parasites within the vector bear equal capabilities to control malaria as well as elimination or indeed eradication.

The use of Insecticide Treated mosquito Nets (ITNs) is currently recommended in Uganda to check on vector spreading the disease. Despite recent fears of resistance to insecticides as well as rebound, insecticide ITNs and minimal indoor residual house spraying (IRS) are still largely recommended to help check on levels of malaria transmission control in Uganda (Killeen, 2013). In order to develop strategies for ownership and use of ITNs, 4 studies were conducted on the outcome to increase the ownership and use of ITNs and both failed to verify the hypothesis "that people who purchase nets will use ITNs more than those who receive them at no cost" (Augustincic Polec et al., 2015 pg 1-6). Instead, a recommendation for educational interventions was made. However, the challenge of the impact of various categories and intensities of education and health promotion still remains at large (Augustincic Polec et al., 2015). One of the Randomized controlled Trial (RCT) conducted in the neighboring Country Tanzania, indicated that IRS is more protective compared to ITNs in Stable transmission zones. Uganda highly relies more on ITNs, which is confirmed to be more protective in unstable transmission zones especial among the rural poor and mass distribution of free ITNs is currently highly preferred. However, the study concluded that there are few well-run trials to quantify the effects of IRS in areas with different malaria transmission (Pluess et al., 2010). In line with the above, current evidence from RCT that compares IRS to IRS also confirmed that IRS bears a great impact on the reduction of malaria incidence in unstable settings. However, the challenge remains that RCT data from such stable malaria settings is quite limited even if it confirms ITN to have better protection than the IRS in unstable malaria settings (Pluess et al., 2010). According to world malaria report 2013, Uganda has high transmission rates of malaria by more than $90 \%$ and ITNs, IRS and Intermittent presumptive treatment in pregnant women are the cornerstone of prevention of the disease in the country (Rica \& Salvador, 2013).

Current evidence shows concern about the cost-effectiveness of malaria control programs. In one of the systemic reviews on cost and cost-effectiveness of malaria control interventions conducted recently revealed an evidence base on donor and domestic allocation of resources 
(White, Conteh, Cibulskis, \& Ghani, 2011). Contrary to Uganda's malaria prevention practices, a recent study conducted in neighboring country Kenya revealed the supremacy of $75 \%$ reduction in risk of transmission at a lower cost USD 9\$ for those sleeping in houses sprayed IRS. However, those using ITNs spend more to a tune USD 29\$ with the lower risk of reduction in transmission of 69\% (Guyatt, Corlett, Robinson, Ochola, \& Snow, 2002).

Currently, it is evident that Artemisinin-based Combination Therapy (ACT) has contributed significantly to the control of malaria in Uganda. Although in 2002 Uganda adopted a Village Health Team strategy that continues to play a key role in the distribution and increasing access to ACT in rural communities, a number of challenges still exist like planning and financial support for transport and storage, local sensitivity tests and establishing a comprehensive quality control systems (Kokwaro, 2009). The current challenge is the fear of history repeating itself like when chloroquine resistance occurred in Uganda in the past and the country resorted to using the drug combinations like Chloroquine and Fansidar (SP). Therefore, plans should be in place to contain resistance to ACTs by developing newer cost effective alternative regimens needed for future use in Uganda and Africa at large (Greenwood, 2014). Most recently, according to WHO world report 2013, Uganda ACTs are free for all ages in the Public sector (WHO, 2013). Furthermore, contrary to the current practice in Uganda, WHO recommends the use of Artesunate Injection in treating children and adults with severe malaria since several trials have shown the drug to have fewer mortalities compared to quinine treatment (Esu, Ee, On, Uwaoma, \& Mm, 2014). The challenge remains in the use of Intravenous Artesunate for public sectors especially the challenge of frequent stock outs this medicine. Hence, the continuous use of Quinine as the second-line antimalarial drug in Uganda Public health sector. This relates to low uptake of ACT in various African countries since most people resort to seeking care from the private sector. The drugs are also quite expensive on retail markets hindering their usage and current call to subsidize their cost is dire (Opiyo, Yamey, \& Garner, 2016).The country also withdrew artemisinin-based monotherapies, meaning the effect of pill burden remains, especially when dealing with Paediatric formulations. Studies on adherence to 24 pills adult dose of coetem (Artemether +Lumefantrine) in the country are limited and future resistance is highly anticipated as few people can actually complete the treatment course of Coetem (Rica \& Salvador, 2013). Since history repeats itself, in the last decade during 2006, WHO highly discouraged treatment of malaria using monotherapy with either chloroquine or Amodiaquine, Uganda continues to discourage ACT monotherapy as well (Mcintosh et al., 2010).

\section{The future challenges for malaria control in uganda}

It is now a year countdown to the 2016-2030 global malaria strategy as well as the idea by Bill \&Melinda Gates Foundation to eliminate the disease by 2040. There remains a lot of international optimism to weather tremendous success is being made by implementing nations since it should ensure that community health workers have everything they need to continue fighting malaria (Foundation, 2018). In line with this report, according to the Uganda Ministry of Health (MOH) hospital survey 2014, there were serious weaknesses and gaps in terms of services availability, financing and in the capacity to offer health services (Health, 2014).

According to Gretchen Newby et al, Rollback of Malaria (RBM) Actions and Investments to eliminate Malaria by 2016-2030 (AIM) as well as WHO Global Technical Strategy for Malaria 2016-2030 (GTS), both supports the idea of elimination and eradication. The current and future recommended WHO package of core interventions towards elimination includes vector control, chemoprevention, diagnostic testing, and treatment. The Same assembly has also recommended the vector control strategy in 1948. Uganda also has a well-established future evidence-based Integrated vector management (IVM) as well (Okia et al., 2016).

The advancement in technology remains a challenge in Uganda as it calls for innovations for future prospects to eradicate malaria. Researchers are likely to develop new efficient technologies like vaccination to control and eradicate the disease. Attempts and trials are 
already ongoing since the last decade on over 40 projects as well as future planned studies in this line including the development of new tools to accompany and complement the Global Action Plans are underway (Access, 2012). One such project is Progress made on RTS, S vaccine effective in preventing a great number of clinical malaria episodes and its progression towards licensing status already justified (Pm, Gelband, Graves, \& Gelband, 2010). One similar study has already suggested that, since the malaria community is considering the main role of a first-generation malaria vaccine to control malaria, it is very important to also strategically document the halted as well as ongoing research projects on the malaria vaccine. This will enable researchers to learn lessons that will be used to support the success of second-generation vaccines in the next decade (Schwartz et al., 2012). Most studies also agree with the call for future improvements in tools to facilitate the achievement of the global ambitious targets. According to Marcel, these global targets to eradicate malaria cannot be achieved with the currently available tools and approaches (Tanner, 2012). In related research, to achieve elimination, we need to strongly strengthen health systems in malaria endemic countries a challenge ahead of Uganda to strengthen her health system capacity to implement the proposed interventions on malaria elimination (de Savignv, 2012). Uganda decentralized her health services resulting in various challenges to the eradication of malaria ranging from poor coordination and capacity building. A related study was done in a neighboring country in Tanzania also revealed that decentralization resulted in weak district coordination and management challenges as well as training its staff (Breman, Alilio, \& Mills, 2004).

In Uganda, to achieve eradication, the private sector must be brought on board. Challenges in overcoming barriers to access and affordability for highly cost effective anti-malarial drugs in the private sector, especially in the rural Uganda are largely in existence. According to Talisuna et al, current data showed that supply-side antimalarial subsidy with intensive communication campaigns greatly improved uptake of ACTs by Uganda's private sector (Talisuna et al., 2012). As the world faces a global challenge of antimalarial resistance, the public sector alone cannot overcome this future challenge. Since recent evidence shows that "Malaria patients worldwide are dependent on the efficacy of ACTs" (Ringwald, Barrette, Rasmussen, \& Newman, 2012). This, therefore, creates a future challenge for Uganda with a rich history of drug resistance to eradicate malaria.

Vector control strategies have been historically proposed with limited success to elimination, especially in Uganda. According to Talisuna, cost-effective interventions require epidemiological evidence and further suggests that such evidence is applied in future business models to fund the malaria control program after 2015 in Uganda (Talisuna, Noor, Okui, \& Snow, 2015). ITNs and IRS are best options recommended for control of transmission of malaria in Sub-Saharan Africa due to the fact that, major vectors of the Anopheles gambiae complex and the An. Funestus, prefer biting humans who are indoors at night (Huho et al., 2012). The looming fear that insecticide-treated mosquito nets (LLINs) will soon get to be less effective against the transmission of malaria is an eminent future challenge that calls for new research on vector control. Recent evidence also shows serious concerns raised on ITNs that are becoming less effective due to insecticide resistance against malaria transmission (Briët, Chitnis, \& Penny, 2012). LLINs destroy sensitive organisms and agents are likely to create evolution towards the emergence as well as the spread of new resistant vectors (Ding, Ubben, \& Wells, 2012). This calls for the need to improve on such approaches and techniques in Uganda. An example of such approaches is evidence from Kenya, where the use of odorbaited traps was proposed to be effective, novel means of integrated malaria control in Kenya by the SolarMal project (Briet, Chitnis, \& Penny, 2012). Furthermore, WHO susceptibility tests also provided a clear indication of the LLINs killing efficacy in semi-field conditions as well. In line with this initiative, options for sustainable malaria control in Uganda are also being recommended in the future, such as integrated vector management (Mutero, Schlodder, Kabatereine, \& Kramer, 2012). Contrary to this new evidence, there exists a new perception raised on prevention of malaria beyond the use of LLINs linked to the lack of systematic, 
consistent, and in-depth health education about malaria. Therefore, the development of personal efficacy to effect the change in their surroundings as well as seeking for appropriate medical care and repellants (Castor, Tuakli, MacLeod, \& Kompala, 2012).Even though Uganda still uses larval source management (LSM) targeting mosquito larvae inside the aquatic habitats as the to eradicate malaria, the approach is less widely practiced as it remains on paper. The future challenge to provide data on the effectiveness of this method still remains despite the WHO reporting the approach as being practiced to control Malaria in Uganda (Tusting et al., 2013). Related evidence confirms that LMS can greatly reduce vector numbers by targeting mosquito larvae aquatic sources. However, randomized crossover trial Conducted in Gambia River, contradicted these findings as it did not cause a reduction in the prevalence of the malaria parasites hence a call for more future studies that challenges such interventions (Tusting et al., 2013).

According to Ambrose, cost-effective interventions require epidemiological evidence and further suggests that such evidence can be applied in the future business models to fund the malaria program after 2015 in Uganda (Talisuna et al., 2015).According to Nicolas et al, in line with this approach, an analysis conducted based on computer simulation models revealed a strong way to identify data gaps hindering rational resource allocation in malaria control programs (Maire, Tarantino, Di Pasquale, Penny, \& Smith, 2012). The need to create linkages between local and international research funders to support malaria elimination in Uganda has also been highlighted and should be further explored (Mshinda, 2012).

Timely treatment of malaria is key in the eradication of the disease. Uganda relies on artemisinin-based drug combinations commonly branded as coetem (Artemether + Lumefantrine). The future challenge lies in the possible development of malaria resistance to artemisinin, hence the need to plan for research on the new generation of antimalarial regimens (Wernsdorfer, 1984). However, the new evidence available, suggests the possibility of future hopes for a single dose cure that will put a relief of pill burden for first line artemisinin-based combinations. The challenge will remain as to how long it will take Uganda to make such interventions affordable, available and accessible to its people. According to Timothy NC Wells, These regimens are already in phase II clinical trials with cutting edge technology capable of overcoming any future emerging malaria resistance leading to elimination (Wells \& Wells T.N.C., 2012). Contrary to this, artemisinin monotherapy has been discouraged in strongest terms, based on the WHO recommendations to prohibit the use of artemisinin monotherapy reportedly used widely in private practice (Greenwood, 2014). Hence balancing the effect of the pill burden against the risk malaria drug resistance is a major challenge for the future scientist in the next 15years. According to WHO world report 2013, Uganda withdrew artemisinin-based monotherapies as well due to fear of resistance.

\section{Discussion}

Findings show that the past challenges in control and eradication of malaria still exist and are likely to last for more than a decade unless measures are in place to mitigate them. Uganda is a low resource country with limited resources to finance malaria eradication programs. The country also has a rampant history of drug resistance by parasites, chronic and persistent antimalarial stock outs in public facilities, unaffordable antimalarial drugs in Uganda's largely private sector, resistance by parasites to vector control interventions like ITNs. Furthermore, Uganda's health facilities largely have very limited capacity in terms of human resource and infrastructure to adequately implement universal parasite-based malaria diagnosis as well as treatment. All these challenges must be addressed if Uganda is to achieve her 2030 international obligations on control and eradication of malaria globally.

Uganda is also likely to face problems in implementing newer effective interventions in the future, due to its financial constraints and lack of political commitment on her health budgets coupled with widespread corruption in the health sector. The past challenges faced in the eradication of malaria ranging from the limited financing of the health sector to drug resistance are still manifest as current challenges in the country, we cannot rule-out such 
Texila International Journal of Public Health

Volume 5, Issue 2, Jun, 2017

challenges like drug resistance in future, and thus, history on malaria control, and eradication challenges may keep repeating itself.

\section{Conclusion}

The historical challenges in the control and eradication of malaria in Uganda are still affecting the current plans to eradicate malaria in the country. Uganda may not eradicate malaria in next 15 years, basing on its historical, current, and future challenges ahead. The country should therefore urgently embark on improving her health infrastructure, build strong human resource network, coordination, as well as operational research in the field of malaria control program. To achieve the 2030 international obligations to control and eradication of malaria will depend on how well Uganda commits to strengthening its health system as well as the Abuja declaration to the allocation of $16 \%$ to the health sector. Lastly, the commitment to the implementation of the global fund meant to control malaria that in past has been widely met with corruption challenges will be a fundamental step in the progress geared towards the control and eradication of malaria in the next 15years.

\section{Acknowledgements}

I would like to acknowledge the efforts of Gabriela Piriz Geneva foundation of medical education and research and Loyda. K. Twinomujuni University of Roehampton and lastly Suma Menon Texila American University Faculty member and Kumar Suresh Texila journal of Public health for Guidance on article Publication

\section{References}

[1]. Access, O. (2012) Challenges in malaria research: Progress towards elimination Malaria surveillance systems: From control to elimination, 11, 1-78.

[2]. Akello, G. (2015). A History of Malaria Control in Uganda: The Limiting Effects of Political and Socio-Economic Inequality in Implementing Global Health Programs. Global Journal of Anthropology Research, 2 (July), 38-49. http://doi.org/10.15379/24102806.2015.02.01.04

[3]. Alilio, M. S., Bygbjerg, I. C., \& Breman, J. G. (2004). Are multilateral malaria researches and control programs the most successful? Lessons from the past 100 years in Africa. American Journal of Tropical Medicine and Hygiene, 71(2 SUPPL.), 268-278.

[4]. Augustincic Polec, L., Petkovic, J., Welch, V., Ueffing, E., Tanjong Ghogomu, E., Pardo Pardo, J., ... Tugwell, P. (2015). Strategies to Increase the Ownership and Use of InsecticideTreated Bed nets to Prevent Malaria. Cochrane Database Syst Rev, 3(6), CD009186. http://doi.org/10.1002/14651858.CD009186.pub2

[5]. Breman, J. G., Alilio, M. S., \& Mills, A. (2004). Conquering the intolerable burden of malaria: What's new, what's needed: A summary. American Journal of Tropical Medicine and Hygiene, 71(2 SUPPL.), 1-15. http://doi.org/71/2_suppl/1 [pii]

[6]. Briet, O. J. T., Chitnis, N., \& Penny, M. (2012).Does mosquito mortality in WHO insecticide susceptibility tests relate to mosquito mortality in LLIN experimental hut studies? Malaria Journal, Conference (Suppl 1), S14. http://doi.org/10.1186/1475-2875-11-S1-O43

[7]. Briët, O. J. T., Chitnis, N., \& Penny, M. (2012). Does mosquito mortality in WHO insecticide susceptibility tests relate to mosquito mortality in LLIN experimental hut studies? 11(Suppl 1), 2-3. http://doi.org/10.1186/1475-2875-11-S1-O43

[8]. Castor, J. P., Tuakli, J., MacLeod, B., \& Kompala, C. (2012). Beyond the nets: Fighting malaria in the 21st century. Malaria Journal, Conference (Suppl 1), S47. http://doi.org/10.1186/1475-2875-11-S1-P74

[9]. Cotter, C., Sturrock, H. J. W., Hsiang, M. S., Liu, J., Phillips, A. A., Hwang, J., ... Gosling, R. D. (2013). The changing epidemiology of malaria elimination: new strategies for new challenges. The Lancet, 382(9895), 900-911. http://doi.org/10.1016/S01406736(13)60310-4

[10].de Savignv, D. (2012). Health systems stewards and health systems researchers: a 
critical partnership for malaria elimination. Malaria Journal, 11 (Suppl 1), O13. http://doi.org/10.1186/1475-2875-11-S1-O13

[11].Ding, X. C., Ubben, D., \& Wells, T. N. C. (2012). A framework for assessing the risk of resistance for antimalarials in development. Malaria Journal, 11(1), 292. http://doi.org/10.1186/1475-2875-11-292

[12].Esu, E., Ee, E., On, O., Uwaoma, A., \& Mm, M. (2014). Artemether for severe malaria (Review). The Cochrane Database of Systematic Reviews, (9), 68. http://doi.org/10.1002/14651858.CD010678.pub2

[13].Foundation, M. G. (2018). The global effort to control malaria is making progress, but funding concerns, weak health. The Lancet Infectious Diseases, 17(1), 28-29. http://doi.org/10.1016/S1473-3099(16)30559-X

[14]. Global technical strategy for malaria 2016-2030. (2016).

[15]. Greenwood, B. (2014). Treatment of Malaria - A Continuing Challenge. New England Journal of Medicine, 371(5), 474-475. http://doi.org/10.1056/NEJMe1407026

[16]. Guyatt, H. L., Corlett, S. K., Robinson, T. P., Ochola, S. A., \& Snow, R. W. (2002). Malaria prevention in highland Kenya: Indoor residual house-spraying vs. insecticide-treated bednets. Tropical Medicine and International Health, 7(4), 298-303. http://doi.org/10.1046/j.1365-3156.2002.00874.x

[17].Health, M. O. F. (2014).Uganda Hospital and Health Centre IV Census Survey 2014.

[18].Hemingway, J., Ranson, H., Magill, A., Kolaczinski, J., Fornadel, C., Gimnig, J. ... Foundation, M. G. (2016). Averting a malaria disaster: will insecticide resistance derail malaria control? 1785-1788. http://doi.org/10.1016/S0140-6736(15)00417-1

[19].Huho, B., Briët, O., Seyoum, A., Sikaala, C., Bayoh, N., Gimnig, J., Killeen, G. (2012). Consistently high baseline estimates for the proportion of human exposure to rural African malaria vector populations that occurred indoors, 11 (Suppl 1), 2875. http://doi.org/10.1186/1475-2875-11-S1-P50

[20]. Kassam, R., Collins, J. B., Liow, E., \& Rasool, N. (2015). A narrative review of the current context of malaria and management strategies in Uganda (Part I). Acta Tropica, 152, 252-268. http://doi.org/10.1016/j.actatropica.2015.07.028

[21]. Killeen, G. F. (2013). A second chance to tackle African malaria vector mosquitoes that avoid houses and don't take drugs. American Journal of Tropical Medicine and Hygiene, 88(5), 809-816. http://doi.org/10.4269/ajtmh.13-0065

[22].Kokwaro, G. (2009). Ongoing challenges in the management of malaria. Malaria Journal, 8Suppl 1(Suppl 1), S2. http://doi.org/10.1186/1475-2875-8-S1-S2

[23].Kyabayinze, D. J., Achan, J., Nakanjako, D., Mpeka, B., Mawejje, H., Mugizi, R. ... Jean-Pierre, V. G. (2012). Parasite-based malaria diagnosis: are health systems in Uganda equipped enough to implement the policy? BMC Public Health, 12(1), 695. http://doi.org/10.1186/1471-2458-12-695

[24]. Maire, N., Tarantino, M., Di Pasquale, A., Penny, M., \& Smith, T. A. (2012). Costeffectiveness of a malaria control programs in sub-Saharan Africa: Analysis of uncertainties using a stochastic individual-based simulation model. Malaria Journal, Conference (Suppl 1), S45-S46. http://doi.org/10.1186/1475-2875-11-S1-P68

[25]. Mcintosh, H., Kl, J., Mcintosh, H., \& Jones, K. L. (2010). Chloroquine or amodiaquine combined with sulfadoxine- pyrimethamine for treating uncomplicated malaria (Review) Chloroquine or amodiaquine combined with sulfadoxine- pyrimethamine for treating uncomplicated malaria, http://doi.org/10.1002/14651858.CD000386.pub2.Copyright

(4),

$10-12$.

[26]. Mshinda, H. (2012). The importance of the linkage between national and international research funders to support malaria elimination. Malaria Journal, 11 (Suppl 1), O4. http://doi.org/10.1186/1475-2875-11-S1-O4

[27]. Mutero, C. M., Schlodder, D., Kabatereine, N., \& Kramer, R. (2012). Integrated vector management for malaria control in Uganda: knowledge, perceptions and policy development. Malaria Journal, 11(1), 21. http://doi.org/10.1186/1475-2875-11-21 
Texila International Journal of Public Health

Volume 5, Issue 2, Jun, 2017

[28].Newby, G., Bennett, A., Larson, E., Cotter, C., Shretta, R., Phillips, A. A., \& Feachem, R. G. A. (2016).The path to eradication: a progress report on the malaria-eliminating countries. The Lancet, 387(10029), 1775-1784. http://doi.org/10.1016/S0140-6736(16)002300

[29]. Okia, M., Okui, P., Lugemwa, M., Govere, J. M., Katamba, V., Rwakimari, J. B. ... Chanda, E. (2016). Consolidating tactical planning and implementation frameworks for integrated vector management in Uganda. Malaria Journal, 1-11. http://doi.org/10.1186/s12936-016-1269-7

[30]. Olowe, O. A., Makanjuola, O. B., Olowe, R. A., Akintola, L., Day, S., \& Hospital, A. (2015). Malaria in Africa and the Historical Perspective: The Journey so, 3(2006), 33-41.

[31]. Opiyo, N., Yamey, G., \& Garner, P. (2016). Subsidizing artemisinin-based combination therapy in the private retail sector (Review) SUMMARY OF FINDINGS FOR THE MAIN COMPARISON, (3).

http://doi.org/10.1002/14651858.CD009926.pub2. www.cochranelibrary.com

[32]. Pluess, B., Fc, T., Lengeler, C., Bl, S., Pluess, B., Tanser, F. C. ... Sharp, B. L. (2010). Indoor residual spraying for preventing malaria (Review), (4). http://doi.org/10.1002/14651858.CD006657.pub2.Copyright

[33].Pm, G., Gelband, H., Graves, P. M., \& Gelband, H. (2010). Vaccines for preventing malaria (pre-erythrocytic) (Review) Vaccines for preventing malaria (pre-erythrocytic), (4). http://doi.org/10.1002/14651858.CD006198.Copyright

[34]. The region, A., Region, E. M., Region, E., \& Region, S. A. (2015).Regional profile.

[35].Rica, C., \& Salvador, E. (2013).World malaria report 2013 countries profile. World Health Organisation.

[36].Ringwald, P., Barrette, A., Rasmussen, C., \& Newman, R. D. (2012).The global challenge of antimalarial drug resistance. Malaria Journal, Conference (Suppl 1), S12. http://doi.org/10.1186/1475-2875-11-S1-O37

[37]. Road, L. (2014). THE UGANDA MALARIA REDUCTION STRATEGIC PLAN 20142020, (May), 1-83.

[38]. Schwartz, L., Brown, G. V, Genton, B., \& Moorthy, V. S. (2012). A review of malaria vaccine clinical projects based on the WHO rainbow table. Malaria Journal, 11(1), 11. http://doi.org/10.1186/1475-2875-11-11

[39]. Talisuna, A. O., Grevval, P., Balyeku, A., Egan, T., Bwire, G., Piot, B. Jagoe, G. (2012). Overcoming the affordability barrier for effective and high-quality life-saving malaria medicines in the private sector in rural Uganda: the Consortium for ACT Private Sector Subsidy (CAPSS) pilot study, 11(Suppl 1), 6-7. http://doi.org/10.1186/1475-2875-11-S1-O16 [40]. Talisuna, A. O., Noor, A. M., Okui, A. P., \& Snow, R. W. (2015). The past, present and future use of epidemiological intelligence to plan malaria vector control and parasite prevention in Uganda. Malaria Journal, 14(1), 158. http://doi.org/10.1186/s12936-015-06774

[41]. Tanner, M. (2012). The science of elimination in practice. Malaria Journal, Conference (Suppl 1), S18. http://doi.org/10.1186/1475-2875-11-S1-O53

[42].Tusting, L. S., Thwing, J., Sinclair, D., Fillinger, U., Gimnig, J., Bonner, K. E. ... Lindsay, S. W. (2013). Mosquito larval source management for controlling malaria. The Cochrane Database of Systematic Reviews, 8(8), CD008923. http://doi.org/10.1002/14651858.CD008923.pub2

[43]. Wells, T. N. C., \& Wells T.N.C. (2012). New drugs for the control and elimination of malaria: A snapshot of the pipeline. Malaria Journal, Conference (Suppl 1), S10. http://doi.org/10.1186/1475-2875-11-S1-O31

[44]. Wernsdorfer, W. H. (1984). Drug-resistant malaria. Endeavor, 8(4), 166-171. http://doi.org/10.1016/0160-9327(84)90080-2

[45]. White, M., Conteh, L., Cibulskis, R., \& Ghani, A. (2011). Costs and cost-effectiveness of malaria control interventions - a systematic review. Malar J, 10(1), 337. http://doi.org/10.1186/1475-2875-10-337 